\title{
Massive stars in low-redshift galaxies
}

\author{
Marie-A. Treyer \\ Laboratoire d'Astrophysique de Marseille, CNRS, \\ Traverse du Siphon, BP 8, F-13376 Marseille Cedex 12, la France
}

\begin{abstract}
I summarize the method commonly used to estimate the current star formation rate per unit volume as a function of redshift from various star formation diagnostics, as well as the often conflicting results from the various multi-wavelength datasets collected in recent years. Combined together, and with the help of theoretical and empirical models, these results allow us to address the issues of excitation, reddening, metallicity, and star formation history in individual galaxies at low redshift. They seem to point to the fact, that a lot of the star formation in the local universe, and potentially at all redshift, occurs in burst mode rather than continuously.
\end{abstract}

\section{Standard star formation diagnostics}

'We are in a situation in which we only measure the tail of the distribution of energy coming from a hot star. The actual beast that lurks shortward of the Lyman limit in only dimly perceived through theoretical modeling of a moving atmosphere, which is an incompletely solved problem', said Conti (1986). Thus we are left with imperfect although numerous ways of tracing massive stars, for example the UV continuum flux shortward of the Lyman limit, the $\mathrm{H} \alpha$ emission line, the $[\mathrm{O}$ II $] \lambda 3727$ forbidden line, the far-IR or the radio continuum fluxes (see Kennicutt 1998 for a review). All these emissions are assumed to be proportional to the current star-formation rate (SFR) of the galaxies. However, converting them into a SFR is model dependent and affected by various poorly understood factors, in particular the amount of light absorbed by dust and re-emitted in the far-IR. The radio flux of a galaxy, though unaffected by dust obscuration, may be contaminated by non stellar emission. Following the footsteps of Lilly et al. (1996) and Madau et al. (1996) in trying to derive the star-formation history (SFH) of the universe, there has been a wealth of SFR estimates at all redshifts based on a variety of diagnostics.

\subsection{Method}

Estimating a SFR per unit volume at a given redshift consists of acquiring a sample of galaxies selected at a massive star sensitive wavelength, of measuring redshifts and estimating spectral types ( $k$-corrections) for the galaxies and computing their luminosity function. The integrated light of the galaxies at the chosen wavelength and mean redshift of the sample is then converted into a SFR 

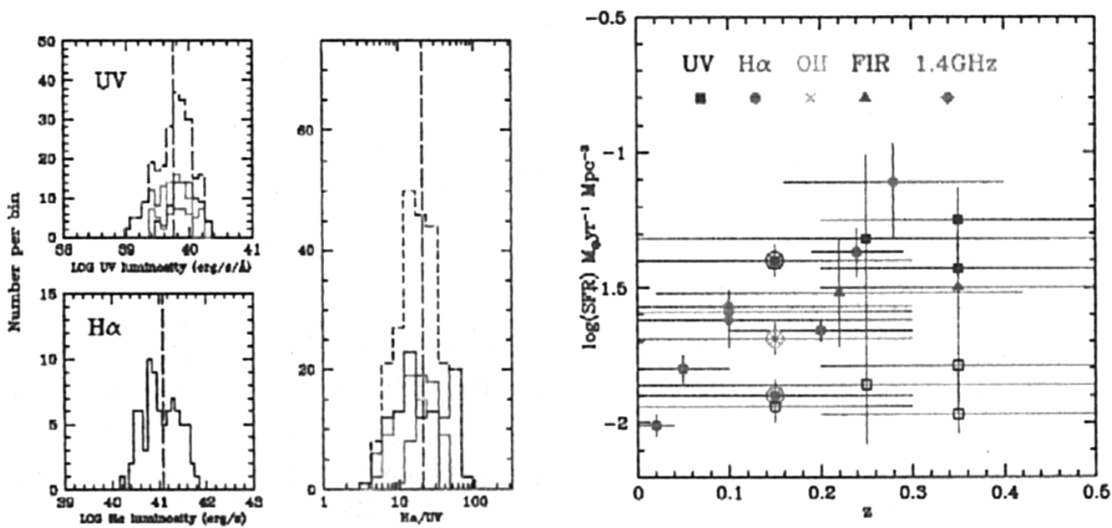

Figure 1. Left: Predicted $\mathrm{H} \alpha$ and UV luminosities as well as $\mathrm{H} \alpha / \mathrm{UV}$ ratio, assuming $S F R=1 \mathrm{M}_{\odot} \mathrm{yr}^{-1}$ and a range of IMF's, upper and lower mass cutoffs, metallicities and ages for the galaxy (Sullivan 2001). The vertical dashed lines shows the canonical values adopted by Kennicutt (1998) assuming a Salpeter IMF with stellar masses in the range $0.1-100 \mathrm{M}_{\odot}$. Right: Compilation of SFR estimates at low redshifts based on UV, $\mathrm{H} \alpha$, [O II], far-IR and $1.4 \mathrm{GHz}$ data as summarized in Table 1 . The empty squares represent the UV-based values uncorrected for dust obscuration.

using a calibration derived from stellar population synthesis models. Short of being able to select galaxies directly at the relevant wavelength, many authors use follow-up measurements of (mostly) optically selected samples.

\subsection{Calibration}

$$
S F R=C \times \mathcal{L} \quad \text { where } \quad \mathcal{L}=\int L \phi(L) \mathrm{d} L
$$

$C$ can be computed from stellar population synthesis models assuming an IMF, stellar tracks and atmospheres, metallicity and, importantly, that the SFR has been constant over the galaxy's lifetime, or at least the last Gyr, so that the light from massive stars has had time to reach a plateau directly proportional to the assumed constant SFR. Figure 1 (left panel) shows the distribution of the $\mathrm{H} \alpha$ and UV luminosities as well as that of the $\mathrm{H} \alpha / \mathrm{UV}$ ratio, assuming $S F R=1 \mathrm{M}_{\odot} \mathrm{yr}^{-1}$ (1/C distribution) and a range of IMF's, upper and lower mass cutoffs, metallicities and ages for the galaxy (Sullivan 2001). The vertical dashed lines show the canonical values adopted by Kennicutt (1998), assuming a Salpeter IMF with stellar masses in the range 0.1-100 $\mathrm{M}_{\odot}$. A parameter not included in these distributions is the fraction of Lyman continuum photons actually absorbed and reprocessed into recombination lines. This fraction is assumed to be $100 \%$, but a lower fraction (as observed in some $\mathrm{H}$ II regions) would lower the expected $\mathrm{H} \alpha$ luminosity for a given SFR. Note the large spread in the conversion factors, even for the $\mathrm{H} \alpha / \mathrm{UV}$ ratio, mostly due to the choice of IMF and time elapsed since the assumed constant star formation began. 
Table 1. Compilation of luminosity densities in $\operatorname{erg~s}^{-1}$ or $\operatorname{erg~s}^{-1} \mathrm{~Hz}^{-1}$ and SFR's at low redshifts. The $C$ factors $(S F R=C \times \mathcal{L})$ are the conversion factors by Kennicutt (1998) assuming a Salpeter IMF with stellar masses between 0.1 and $100 \mathrm{M}_{\odot}$. The last column lists the logarithmic values of the SFR in units of $h \mathrm{M}_{\odot} \mathrm{yr}^{-1} \mathrm{Mpc}^{-3}$ where $h=\mathrm{H}_{0} / 50 \mathrm{~km} \mathrm{~s}^{-1} \mathrm{Mpc}^{-1}$.

\begin{tabular}{lllllll}
\hline \hline reference & $\lambda$ & $\Delta z$ & $\log \mathcal{L}$ & $\pm \operatorname{dlog} \mathcal{L}$ & $\log C$ & $S F R$ \\
\hline Pascarelle et al. 98 & $1500 \AA$ & $0-0.5$ & 25.99 & $0.31 /-0.22$ & -27.85 & -1.86 \\
Treyer et al. 98 & $2000 \AA$ & $0-0.3$ & 25.91 & $0.06 /-0.06$ & -27.85 & -1.94 \\
Wilson et al. 02 & $2500 \AA$ & $0.2-0.5$ & 26.06 & $0.12 /-0.12$ & -27.85 & -1.79 \\
Lilly et al. 96 & $2800 \AA$ & $0.2-0.5$ & 25.88 & $0.07 /-0.07$ & -27.85 & -1.97 \\
Gallego et al. 95 & $\mathrm{H} \alpha$ & $0-0.04$ & 39.09 & $0.04 /-0.04$ & -41.10 & -2.01 \\
Tresse \& Maddox 98 & $\mathrm{H} \alpha$ & $0.1-0.3$ & 39.44 & $0.04 /-0.04$ & -41.10 & -1.66 \\
Gronwall et al. 02 & $\mathrm{H} \alpha$ & $0-0.1$ & 39.30 & $0.05 /-0.05$ & -41.10 & -1.80 \\
Pascual et al. 01 & $\mathrm{H} \alpha$ & $0.2-0.3$ & 39.73 & $0.09 /-0.09$ & -41.10 & -1.37 \\
Sullivan et al. 00 & $\mathrm{H} \alpha$ & $0-0.3$ & 39.20 & $0.06 /-0.06$ & -41.10 & -1.90 \\
Hammer et al. 97 & {$[\mathrm{O} \mathrm{II]}$} & $0.2-0.5$ & 38.63 & $0.06 /-0.08$ & -40.85 & -2.22 \\
Sullivan et al. 00 & {$[\mathrm{O} \mathrm{II}]$} & $0-0.3$ & 39.16 & $0.06 /-0.06$ & -40.85 & -1.69 \\
Flores et al. 99 & $8-10^{3} \mu \mathrm{m}$ & $0.2-0.5$ & 41.88 & $0.25 /-0.25$ & -43.34 & -1.46 \\
Mann et al. 02 & $8-10^{3} \mu \mathrm{m}$ & $0-0.43$ & 41.82 & $0.20 /-0.20$ & -43.34 & -1.52 \\
Haarsma et al. 00 & $1.4 \mathrm{GHz}$ & $0 .-0.4$ & 26.75 & $0.14 /-0.21$ & -27.86 & -1.11 \\
Mobasher et al. 99 & $1.4 \mathrm{GHz}$ & $0-0.3$ & 26.27 & $0.07 /-0.07$ & -27.86 & -1.59 \\
Serjeant et al. 02 & $1.4 \mathrm{GHz}$ & $0-0.3$ & 26.24 & $0.10 /-0.10$ & -27.86 & -1.62 \\
Sadler et al. 02 & $1.4 \mathrm{GHz}$ & $0-0.3$ & 26.29 & $0.06 /-0.09$ & -27.86 & -1.57 \\
\hline \hline
\end{tabular}

\subsection{Dust corrections}

The UV continuum of a galaxy and the strength of its $\mathrm{H} \alpha$ line can be strongly diminished by dust obscuration. The far-IR flux is also directly dependent on the amount of light absorbed by dust. It is therefore crucial to estimate this quantity. The cosmic far-IR background exceeds the optical+UV background, suggesting that more than $50 \%$ of the light emitted by massive stars at all redshifts is absorbed by dust and re-emitted in the far-IR (Gispert, Lagache \& Puget 2000). Various theoretical and empirical methods can be used to quantify the effects of dust opacity, which seems to be not only morphology-, but also luminosity/SFR-dependent (see review by Calzetti 2001).

\section{The ultraviolet}

As UV photons coming from sufficiently high redshift fall into the optical window, the UV has been the preferred wavelength to select high-redshift galaxies via the Lyman-break technique. Although there exist several instruments with UV imaging or spectroscopic capabilities to study local galaxies, the only instrument combining a wide field with sufficient sensitivity suitable for survey mode has been the balloon-borne FOCA telescope (Milliard et al. 1992). The FOCA 
$2000 \AA ̊$ images allowed us to apply the above technique to local galaxies in a way that is directly comparable to the high-redshift universe.

\subsection{The FOCA sample}

The spectroscopic follow-up of a magnitude limited sample of $\sim 270$ FOCA galaxies, the $k$-corrections, stellar absorption corrections, and dust extinction corrections applied to these galaxies, and the subsequent estimates of the luminosity function and SFR are described in Treyer et al. (1998) and Sullivan et al. (2000). In brief, we find that FOCA can detect galaxies in the UV to a redshift of $z \simeq 0.3$ with a mean $z \simeq 0.15$ at the magnitude limit of our sample $\left(m_{2000 \AA}<20.70 \mathrm{mag}\right.$ in the AB system). The flux calibrated optical spectra obtained with the multi-fiber spectrograph WYFFOS on the WHT allowed us to measure the strength of several emission lines, in particular $\mathrm{H} \alpha$ and $[\mathrm{O} \mathrm{II}]$. We use the observed Balmer decrements and an empirical extinction curve (Calzetti et al. 2000) derived from starburst galaxies to correct the emission lines and UV continuum for the effects of dust obscuration. We also obtained $1.4 \mathrm{GHz}$ fluxes with the VLA for a subsample of the galaxies, allowing us to estimate and compare SFR's based on four different star-formation diagnostics (Sullivan et al. 2001).

\subsection{Low redshift star-formation rates}

Figure 1 (right panel) shows a compilation of SFR estimates at low redshift, based on a variety of surveys summarized in Table 1 and assuming the conversion factors from light to SFR proposed by Kennicutt (1998). The UV, $\mathrm{H} \alpha$ and [O II] based estimates derived from the UV-selected FOCA sample are circled. All UV estimates are corrected upward by $1.4 \mathrm{mag}$ to account for dust obscuration. This value is based on our own estimate in the FOCA sample using Calzetti et al. (2000)'s extinction law.

It is clear from Figure 1 (right panel) that the value of the local SFR is still very much uncertain. Not only SFR estimates differ from one survey to the next, but our survey shows inconsistent results between $\mathrm{H} \alpha$ and UV-derived values for the same galaxies. There are a number of reasons for the discrepancies between diagnostics: different selection criteria probe different populations, the various conversion factors are not only model dependent, they also vary differently from one another with different parameters (Figure 1 left), and there are large uncertainties in dust correction in each surveys. Charlot \& Longhetti (2001) also showed, that neglecting the absorption by dust in $\mathrm{H}$ II regions and the contamination of emission lines by stellar absorption can strongly underestimate $\mathrm{H} \alpha$ estimates. Before resolving the discrepancy between the various surveys, it is crucial to understand the discrepancy within our own sample and therefore to look at individual galaxies, rather than integrated light.

\section{Erratic star-formation rate}

One major assumption, when computing conversion factors between light and SFR, is that star formation is constant or at least varying smoothly, which is probably justified when considering a well-behaved, more quiescent population 

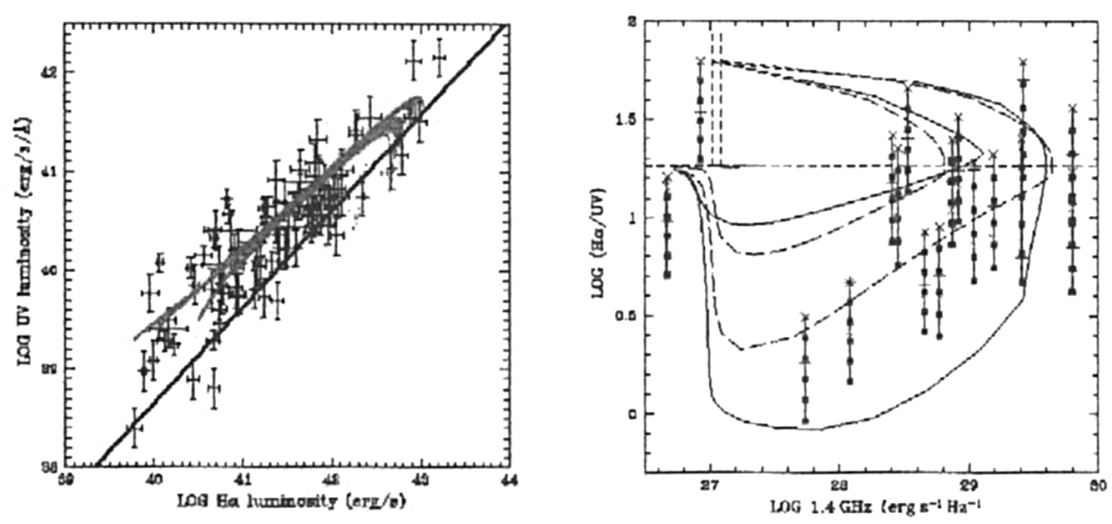

Figure 2. Left: UV vs. $\mathrm{H} \alpha$ luminosities for our UV-selected sample of galaxies. The curved lines show the predicted paths of galaxies undergoing a series of starbursts of various strengths superimposed on a continuous SFH (Sullivan et al. 2000). Right: $\mathrm{H} \alpha / \mathrm{UV}$ as a function of $1.4 \mathrm{GHz}$ luminosities. The vertical tracks and symbols span a range of dust corrections while the horizontal dashed line shows the $\mathrm{H} \alpha$ /UV ratio expected for a constant SFR (Sullivan et al. 2001). The loops are bursting models as in left panel.

such as an optically selected sample. Figure 2 shows that UV-selected galaxies appear not to have such simple lives.

\subsection{UV versus $\mathrm{H} \alpha$ versus radio}

Figure 2 (left panel) shows UV vs. $\mathrm{H} \alpha$ luminosities for our UV-selected sample of galaxies. The straight line represents the constant SFR prediction assuming the canonical value by Kennicutt (1998). Varying the IMF or the amount of dust extinction will move this line higher or lower, a SFR-dependent dust correction will improve the slope of the correlation (Sullivan et al. 2001), but none of these parameters seems able to explain the observed scatter. A natural way to do so is to allow galaxies more complex SFH's. The bundle of curved lines shows the predicted paths of galaxies undergoing a series of starbursts of various strengths superimposed on a continuous SFH (Sullivan et al. 2000). As a starburst breaks out, both the $\mathrm{H} \alpha$ and UV luminosities are boosted towards the top right corner of the plot. When the burst ends, the UV and $\mathrm{H} \alpha$ luminosities decrease although slower for the UV continuum as it originates from stars with a wider range of main sequence lifetimes $(\lesssim 100 \mathrm{Myr}$ for the UV vs. $\sim 20 \mathrm{Myr}$ for stars contributing to the $\mathrm{H} \alpha$ flux).

Figure 2 (right panel) shows $\mathrm{H} \alpha / \mathrm{UV}$ as a function of $1.4 \mathrm{GHz}$ luminosity, obtained for a small subsample of our galaxies (Sullivan et al. 2001). The radio flux is insensitive to dust and assumed proportional to the SFR. The vertical tracks and symbols span a range of dust corrections including luminosity dependent estimates, while the horizontal dashed line shows the $\mathrm{H} \alpha / \mathrm{UV}$ ratio expected for a constant SFR assuming the canonical calibration. Again, even complex dust corrections seem unlikely to explain the scatter, while erratic SFH's (the loops in Figure 2, right panel) provide a straightforward solution. 

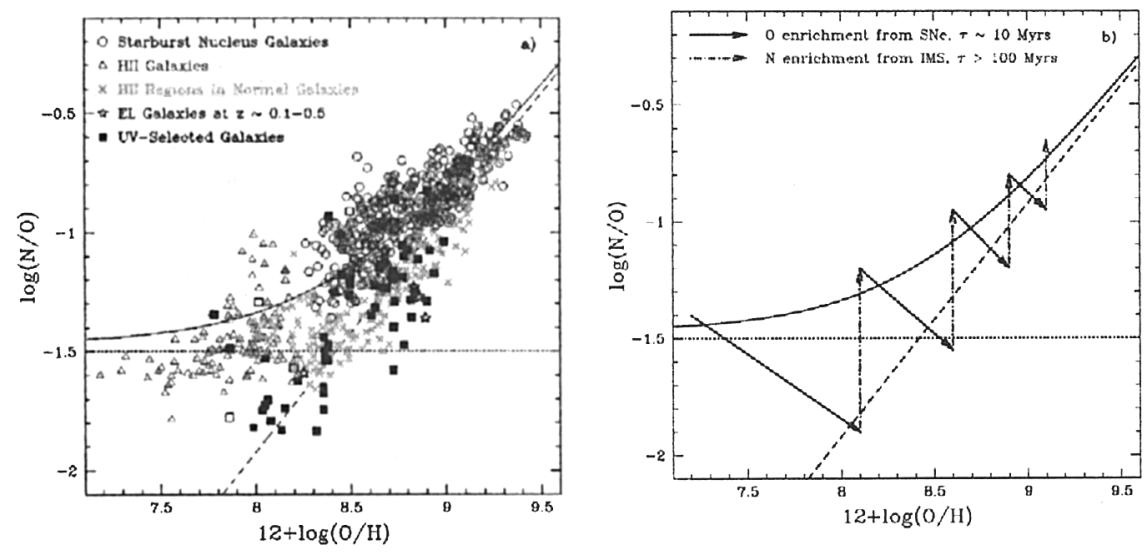

Figure 3. Left: $\mathrm{N} / \mathrm{O}$ abundance ratio as a function of $\mathrm{O} / \mathrm{H}$ for the UVselected galaxies (squares) and various comparison samples. Theoretical curves for a primary (dotted line), a secondary (dashed line), and a primary + secondary (solid line) production of nitrogen are shown. Right: Evolution model of $\mathrm{N} / \mathrm{O} v s$. $\mathrm{O} / \mathrm{H}$ assuming a sequence of starbursts separated by quiescent periods. This scenario assumes that each burst first produces oxygen enrichment due to massive star evolution on short time-scales $(\tau \approx 10 \mathrm{Myr})$, followed by significant nitrogen enrichment on longer time-scales $(\tau>100 \mathrm{Myr})$ due to intermediate-mass star evolution.

\subsection{Chemical abundances}

Independent evidence for discontinuous SFH's is found from chemical abundances. We were able to measure $\mathrm{N} / \mathrm{O}$ and $\mathrm{O} / \mathrm{H}$ abundance ratios for a subsample of UV-selected galaxies and found that a fraction of them exhibits lower N/O ratios at a given oxygen abundance than predicted from primary and secondary nitrogen production models (Figure 3, Contini et al. 2002). This again may be explained by a series of bursts separated by quiescent periods. The dispersion in $\mathrm{N} / \mathrm{O}$ could be due to a delayed release of nitrogen produced in low-mass longerlived stars, compared to oxygen produced in massive, short-lived stars (Henry et al. 2000). During starburst, $\mathrm{O} / \mathrm{H}$ increases as the most massive stars begin to die and supernovae release oxygen into the ISM. A few tens of Myrs after the burst, the massive $\mathrm{O}$ stars producing oxygen will be gone, at which point $\mathrm{N} / \mathrm{O}$ should be minimal with a value roughly limited by the yields of $O$ and secondary $\mathrm{N}$ from the massive stars. Then $\mathrm{N} / \mathrm{O}$ will rise again as intermediate-mass stars begin to contribute to the primary and secondary production of nitrogen, approaching a maximum value limited by the yields of $\mathrm{N}$ and $\mathrm{O}$ from all stars. A galaxy undergoing successive starbursts will thus oscillate between these two limits of N/O, as illustrated in Figure 3, right panel (Contini et al. 2002).

\section{Conclusion}

It appears from the large scatter in the various SFR estimates at low redshift that a lot of work remains to be done to understand large scale star formation 
in even nearby galaxies. In particular, the conversion from observed light to an underlying SFR needs more complex modeling than commonly used. Quantifying dust obscuration is a major task among other things. Equally important is the fact that a lot of the star formation in the local universe, and potentially at all redshift, seems to occur in burst mode rather than continuously. This hypothesis is supported by UV, $\mathrm{H} \alpha$ and radio observations of a well defined sample of UV-selected galaxies, and independently by the measurement of their chemical abundances. Similar results are found in galaxies selected by their $\mathrm{H} \alpha$ emission (Gil de Paz et al. 2000) and in the infrared (Elbaz, private communication). Multi-wavelength observations of larger samples of galaxies, selected at wavelengths where massive stars dominate the flux, will help to probe further into star and dust formation on the scale of whole galaxies.

\section{References}

Calzetti, D. 2001, PASP 113, 1449

Calzetti, D., Armus, L., Bohlin, R.C., Kinney, A., et al. 2000, ApJ 533, 682

Charlot, S., Longhetti, M. 2001 MNRAS 323, 887

Conti, P.S 1986, in: C.W.H. de Loore, A.J. Willis \& P. Laskarides (eds.), Luminous Stars and Associations in Galaxies, Proc IAU Symp. No 116 (Dordrecht: Reidel), p. 239

Contini, T., Treyer, M.-A., Sullivan, M., Ellis, R.S. 2002, MNRAS 330, 75

Flores, H., Hammer, F., Thuan, T. X., et al. 1999, ApJ 517, 148

Gallego, J., Zamorano, J., Aragón-Salamanca, A., Rego, M. 1995, ApJ (Letters) 455, L1

Gil de Paz, A., Aragón-Salamanca, A., Gallego, J., Alonso-Herrero, A., et al. 2000, MNRAS 316, 357

Gispert, R., Lagache, G., Puget, J.L. 2000, A\&A 360, 1

Gronwall, C. 1999, in: S. Holt \& E. Smith (eds), After the Dark Ages: When Galaxies were Young (AIP Press), p. 335

Haarsma, D., Partridge, R., Windhorst, R., Richards, E. 2000, ApJ 544, 641

Hammer, F., Flores, H., Lilly, S.J., Crampton, D., et al. 1997, ApJ 481, 49

Henry, R.B.C, Edmunds, M.G, Köppen, J. 2000, ApJ 541, 660

Kennicutt, R. 1998, Ann. Review Astron. Astrophys. 36, 189

Lilly, S.J., Le Fèvre, O., Hammer, F., Crampton, D. 1996, ApJ (Letters) 460, L1

Madau, P., Ferguson, H.C., Dickinson, M., Giavalisco, M., et al. 1996, MNRAS 283, 1388

Mann, R., Oliver, S., Carballo, R., Franceschini, A., et al. 2002, MNRAS 332, 549

Milliard, B., Donas, J., Laget, M., Armand, C., Vuillemin, A. 1992, A\&A 257, 24

Mobasher, B., Cram, L., Georgakakis, A., Hopkins, A. 1999, MNRAS 308, 45

Pascarelle, S.M., Lanzetta, K.M., Fernández-Soto, A. 1998, ApJ (Letters) 508, L1

Pascual, S., Gallego, J., Aragón-Salamanca, A., Zamorano, J. 2001, A\&A 379, 798

Sadler, E., Jackson, C., Cannon, R., McIntyre, V., et al. 2002, MNRAS 329, 227

Serjeant, S., Gruppioni, C., Oliver, S. 2002, MNRAS 330, 621

Sullivan, M. 2001, PhD thesis, University of Cambridge, UK

Sullivan, M., Mobasher, B., Chan, B., Cram, L., et al. 2001, ApJ 558, 72

Sullivan, M., Treyer, M.-A., Ellis, R.S., Bridges, T., et al. 2000, MNRAS 312, 442 
Tresse, L., Maddox, S.J. 1998, ApJ 495, 691

Treyer, M.-A., Ellis, R.S., Milliard, B., Donas, J., Bridges, T. 1998, MNRAS 300303

Wilson, G., Cowie, L., Barger, A., Burke, D. 2002, AJ 124, 1258

\section{Discussion}

OEY: A number of authors (including Clarke \& Oey 2002) have suggested, that the escape of ionizing photons depends on the morphology of the ISM. We have suggested that the escape fraction is large, when the cool ISM has been surrounded by high star formation. Have you been able to investigate these kinds of possibilities, for example by normalizing the UV luminosities by galaxy $\mathrm{M}_{B}$ or some mass indicator?

TREYER: We have explored models with an escape fraction of ionizing photons as large as $30 \%$. It does help bringing the SFR estimated from $\mathrm{H} \alpha$ a bit closer to that derived from the UV luminosity. However, to account for the large scatter in the UV/H $\alpha$ relation, most of the galaxies would need an escape fraction much larger than $30 \%$. Until recently, we didn't have reliable $B$-band magnitudes or any other mass indicator. Now that we have good $B$-band photometry, we can certainly explore your suggestion.

WALBORN: Resolved local HII regions show that the optical and UV continua are dominated by a 3-4 Myr-old population, that has already evacuated its immediate surroundings, while the nebulae are ionized by a younger, 1-2 Myr year old generation that has a higher extinction. This dichotomy may be related to the observation, that the extinction derived from the Balmer decrement of starbursts is always higher than that from the UV continuum. I wonder, if it could also be related to the higher apparent SFR you find from the UV continuum, as compared to the nebular lines.

TREYER: Actually, the SFR derived from the UV continuum would agree with that derived from $\mathrm{H} \alpha$ if we did not correct the UV at all, but this seems unphysical. And even if the UV luminosities were totally unobscured by dust, this would still not explain the large scatter in the UV/H $\alpha$ correlation. There remain many galaxies with uncorrected UV luminosities much higher than expected from a constant SFR scenario for a given $\mathrm{H} \alpha$ luminosity.

LEITHERER, comment on Walborn's comment: The UV continuum in the spectrum of an integrated stellar population forming at a constant rate comes from late $\mathrm{O}$ - to early B-type stars, not from early O-type stars. There are simply many more B-type stars then O- type stars. O-type stars are only seen in the wind P-Cygni lines. This refers to continuous star formation, as applicable to galaxies observed through a large aperture. 